\title{
Accounting meets Finance
}

\author{
Bericht zum wissenschaftlichen Private Equity-Kolloquium mit \\ Professor Josh Lerner in Hamburg
}

Erstmalig lud der Bundesverband Deutscher Kapitalbeteiligungsgesellschaften (BVK e.V.) am 24. Juni 2010 zu einem exklusiven Private Equity-Kolloquium in die Bucerius Law School nach Hamburg ein, an dem Professoren und Doktoranden verschiedener Controlling- und Finance-Lehrstühle teilnahmen. Ziel des Kolloquiums war ein Austausch der deutschen Wissenschaftler mit Praktikern der Branche sowie mit einem der weltweit renommiertesten Private Equity-Forscher, Professor Josh Lerner von der Harvard Business School.

Zunächst stellten fünf deutsche Wissenschaftler aktuelle Forschungsprojekte vor, die sich aus verschiedenen Perspektiven mit privatem Beteiligungskapital beschäftigen. So berichtete Professor Kaserer (TU München) von seinen Erkenntnissen zu „Net Asset Value Discounts in Listed Private Equity Funds“. In diesem Zusammenhang wurde einerseits die generelle Frage nach der Marktbewertung von Private Equity gestellt. Andererseits ging es darum festzustellen, inwiefern ein Unterschied zwischen den buchhalterischen Vermögenswerten und der Marktbewertung von Private Equity besteht. André Gill (Goethe-Universität Frankfurt a.M.) beschäftigte sich mit den Vorteilen privater Eigentümerschaft durch Private Equity im Gegensatz zu gelisteten Unternehmen. Er stellte auf Basis verschiedener Kennzahlen des Rechnungswesens eine signifikant bessere Performance der Unternehmen in privater Trägerschaft fest und konnte daraus Implikationen für die Unternehmensführung und das Controlling ableiten. Nils Middelberg (Universität Duisburg-Essen) arbeitete Faktoren des Fundraisings von Venture Capital heraus. Als zentrale Aspekte mit positiver Wirkungsrichtung wurden die wahrgenommene Vertrauenswürdigkeit („Trustworthiness") sowie wahrgenommene Steuerbarkeit („Controllability“) identifiziert. Mit der Mittelausstattung junger Unternehmen im Biotechnologiemarkt beschäftigte sich Stefanie Hochhold (LMU München). Sie betonte die fallbezogene Bedeutung von externer Managementerfahrung und dem Zugang
Tagungsort: Bucerius Law School in Hamburg

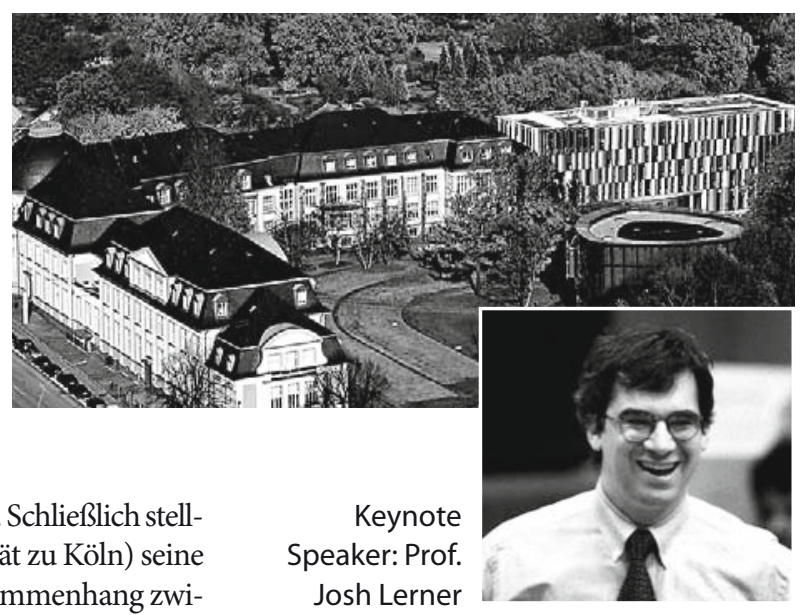

zu technischem Know-how. Schließli te Niklas Hüther (Universität zu Köln) seine Untersuchungen zum Zusammenhang zwischen Managementverhalten und der Effektivität von Vergütungsstrukturen vor.

Im Anschluss an die Vorträge aus Deutschland ging Professor Lerner auf die zentralen Trends der internationalen Private Equity-Forschung ein. Er eröffnete seine Ausführungen mit der divergierenden Wahrnehmung von Wissenschaft („Superior Governance Form") und breiter Öffentlichkeit („Challenge to Financial Stability“). Darauf aufbauend stellte er seine Sicht der derzeitigen Regulierungsdiskussion innerhalb der EU dar. Anschließend ging er detailliert auf drei seiner eigenen aktuellen Studien ein, die sich um die auch in Deutschland intensiv diskutierten Themenschwerpunkte drehen: Mitarbeitereffekte, Innovationstätigkeit und makroökonomische Wirkungen von Private Equity.

Noch interessanter als die beeindruckenden Untersuchungen fanden die deutschen Wissenschaftler den Ausblick, in dem Professor Lerner auf künftigen Forschungsbedarf einging. In diesem Zusammenhang merkte er an, dass 98 Prozent der Studien auf US-amerikanischen Daten beruhen und die Wirkung der institutionellen Ausgestaltung auf Private Equity somit offen ist.

Die abschließende Diskussion wurde von Dr. Hanns Ostmeier (Präsident des BVK) moderiert und diente insbesondere dem Austausch von wissenschaftlicher und praktischer Perspektive. In diesem Zusammenhang wurde angemerkt, dass die Wissenschaft sich vielfach auf die „klassische“, also Finanz- und controllingorientierte For- schung zu Private Equity beschränke. Die Wahrnehmung von Private Equity in der Öffentlichkeit sei zumindest ebenso relevant, insbesondere vor dem Hintergrund der im deutschen Mittelstand vielfach bestehenden Nachwuchsproblematik. Hier wurde nicht nur die Aktivität verschiedener betriebswirtschaftlicher Disziplinen, sondern auch ein interdisziplinärer Ansatz erwogen.

Dabei wurde auch auf die in Zusammenarbeit mit PricewaterhouseCoopers entwickelte „Private Equity Database“ verwiesen, die interessierten Forschern komplette Jahresabschluss- und Transaktionsdaten großer deutscher Leveraged Buyouts (LBO) zur Verfügung stellt. Die Datenbank betrachtet der BVK als wichtige Maßnahme zur Verbesserung der Transparenz der Private Equity-Branche. Erste Studien auf Grundlage der Datenbank zur Performance deutscher LBOs und zu Aspekten der Unternehmensbesteuerung liegen zwar bereits vor, allerdings wünscht sich der BVK eine noch aktivere Nutzung.

Aufgrund der sehr positiven Wahrnehmung der Veranstaltung wurde von den anwesenden Wissenschaftlern angeregt, einen Gedankenaustausch zwischen nationalen und internationalen Wissenschaftlern sowie der Praxis im Bereich Private Equity zu institutionalisieren.

Thorsten Knauer und Friedrich Sommer, Münster 